\title{
Comparison on space charge and voltage distribution of high voltage insulator subjected to different contamination levels
}

\author{
N. A. Samuri ${ }^{1}$, N. A. Othman ${ }^{2}$, M. A. M. Piah ${ }^{3}$, N. A. M. Jamail ${ }^{4}$, H. Rosli ${ }^{5}$ \\ ${ }^{1,2,4,5}$ Department of Electrical Power Engineering, Faculty of Electrical \& Electronic Engineering, \\ Universiti Tun Hussein Onn Malaysia (UTHM), Malaysia \\ ${ }^{3}$ Institute of High Voltage and High Current, School of Electrical Engineering, \\ Universiti Teknologi Malaysia (UTM), Malaysia
}

\begin{tabular}{l}
\hline \hline Article Info \\
\hline Article history: \\
Received Jan 2, 2019 \\
Revised Mar 11, 2019 \\
Accepted Mar 29, 2019 \\
\hline
\end{tabular}

Keywords:

Space charge

Transmission line

Glass insulators

Porcelain insulators

Voltage distribution

\begin{abstract}
This paper presents the study of space charge distribution on high voltage (HV) insulators under different levels of contamination. Two types of HV insulators were used in this work particularly glass and porcelain insulators. A string of 4-unit glass and porcelain insulators with $33 \mathrm{kV}$ of lines voltage was designed and simulated using Quickfield ${ }^{\mathrm{TM}}$ software. Four levels of contamination layer with different thickness have been applied on the surface of insulators to observe the effect of space charge distribution. Simulation results show that different types of insulators used at transmission lines give different effects on charge and voltage distribution. It is also found that the amplitude of charge for a single porcelain insulator is much higher compared to a single glass insulator. Similarly for a string of 4-unit insulators, the voltage distribution along the creepage distance of porcelain insulators is much higher compared to glass insulators under all contamination levels.
\end{abstract}

Copyright $\odot 2019$ Institute of Advanced Engineering and Science. All rights reserved.

\section{Corresponding Author:}

Nordiana Azlin binti Othman,

Department of Electrical Power Engineering,

Universiti Tun Hussein Onn Malaysia (UTHM),

86400-Batu Pahat, Johor, Malaysia.

Email: ndiana@uthm.edu.my

\section{INTRODUCTION}

High voltage (HV) insulators particularly glass and porcelain play a significant role in electric power transmission system. In transmission and distribution, HV insulators are positioned between two towers or pole body to avert any undesirable current flow to the earth [1-2]. HV insulators mounted at power lines are exposed to the atmosphere tend to accumulate the contamination on the insulation surface. The contaminated insulators can be said no problem in dry conditions. However, contaminated insulators in wet conditions may threaten the performance of insulator which may lead to the insulation degradation [3]. The accumulation of contamination sources on the surface of insulator is believed may attract space charge and hence modify the local field distribution [4].

One of the concerns of these insulators is the existence of the space charge on their surfaces. Generally, space charge can be said as a collection of particles with a net electric charge occupying a region, either in free space or in a device. According to [5], all charged carriers including electrons, holes, charged particles or ions, which can exist within the dielectric material, trapped by or transported through the material under the application of external field are also known as space charge.

An inherent nature of insulator that tends to accumulate the trapped electrical charges within the insulation material has led the space charge existence. The presence of space charge at any region of insulator is certainly distort the original electric field distribution and may result in the enhancement of local electric fields to the highest level over a long period of time. This situation would then leads to the premature failure 
of insulating material due to the degradation process which can lead to electrical breakdowns and electrostatic discharges [6]. It is proven that the hetero space charges are always observed before breakdown occurs [7]. Under the higher applied electric fields at the higher temperature, a large amount of hetero space charges was detected earlier after the voltage application [8]. In addition, more charges are found trapped in the bulk of an aging sample after a long time of electrical aging time [9].

Literature studies on space charge not only limited to experimental studies but also simulation studies [10-12]. The uneven distribution of voltage and electric field along a sting insulator found in [13] is due to the presence of coupling capacitance amongst discs of the insulator string. The charge distribution on the glass insulator surfaces was investigated using QuickField ${ }^{\mathrm{TM}}$ Professional software in [14] shows the polarity of charge swinging occurs at the centre of the suspension string insulator of I-type. Meanwhile in [15], it is revealed that the presence of contamination affects the charge distribution of the insulator located near to the ground electrode in glass insulator string.

Based on the aforementioned studies, the charge distribution on the surface of porcelain insulators has not yet being investigated; which provide a substantial area for researchers to explore more on the distribution of charge. Therefore, this paper proposes a study on the voltage and space charge distribution on $\mathrm{HV}$ glass and porcelain insulators subjected to different levels of contamination.

\section{MODELLING DESIGN}

The modelling of glass and porcelain insulators was conducted using finite element software of QuickField $^{\mathrm{TM}}$. The electrostatic problem type was chosen due to the variation of capacitive systems such as fuses, transmission lines and so forth can be analysed. Furthermore, in the electrostatic problem type, voltages, electric fields, space charges, capacitances, and electric forces can be extracted and examined. By using this software, the space charge is calculated using Gauss Law. According to the Gauss theorem, total electric charge in a particular volume can be calculated as a flux of electric displacement over its closed boundary as:

$$
q=\oint_{S}(D \cdot n) d s
$$

Cap and pin suspension glass of U100BL type [16] and porcelain ANSI 52-3 type [17] are selected as the main research object in this work. These insulators are designed according to their cross-section diagram as depicted in Figure 1. It is noticed from Figure 1 that there are five separate regions for both insulators is needed to be modelled for a single unit of insulators with the supply voltage of $11 \mathrm{kV}$. The string of four insulators is subsequently modelled in free space with the supply voltage of $33 \mathrm{kV}$ is applied to the fourth insulator (nearest to HV line) while the first insulator (top insulator cap) is grounded.

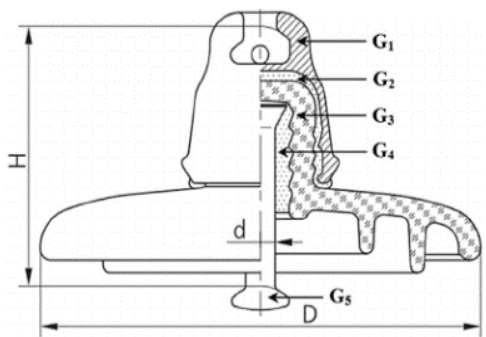

(a)

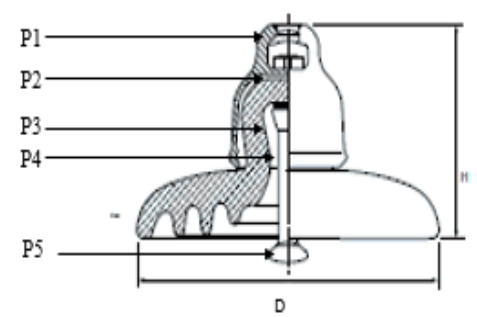

(b)

Figure 1. Cross-sectional diagram of, (a) glass, (b) porcelain insulator

The relative electric permittivity of glass and porcelain insulator are tabulated in Table 1 and Table 2, respectively was manually inserted into the software. Since the insulator has a symmetrical shape, this simulation process is conducted in an axisymmetric 2D model class. For the modelling of the contaminated insulator, a thin film with a different thickness of contamination layer listed in Table 3 was created. By adopting the properties of sea water as the contamination source, this film was modelled as a uniform layer on the entire surface of the insulator. 
Table 1. Material properties of glass insulator [15]

\begin{tabular}{cc}
\hline Types of material & Relative Electric Permittivity \\
\hline Cement (G2 \& G4) & 15 \\
Glass (G3) & 4.2 \\
Cast Iron (G1\&G5) & 1000 \\
Air & 1 \\
\hline
\end{tabular}

Table 2. Material properties of porcelain insulator [18]

\begin{tabular}{cc}
\hline Types of material & Relative Electric Permittivity \\
\hline Cement (P2 \& P4) & 2 \\
Porcelain (P3) & 6 \\
Metal (P1\&P5) & 1000 \\
Air & 1 \\
\hline
\end{tabular}

Table 3. Contaminations level of insulators [19]

\begin{tabular}{cc}
\hline Level of Contamination & Thickness $(\mathrm{mm})$ \\
\hline No contamination (clean) & $0 \mathrm{~mm}$ \\
Light contamination & $1.0 \mathrm{~mm}$ \\
Medium contamination & $1.5 \mathrm{~mm}$ \\
Heavy contamination & $2.0 \mathrm{~mm}$ \\
\hline
\end{tabular}

\section{RESULTS AND ANALYSIS}

The voltage and charge distribution results presented in this section were obtained based on contour plot that measured along the insulator creepage distance under different levels of contamination conditions. The results presented in this section are started with a single unit of insulator followed by a string of four unit insulators.

\subsection{Voltage distribution for single glass insulator}

Figure 2 shows the voltage distribution contour plot for a single glass insulator in clean and contaminated conditions. It is obvious from the Figure 2 (a) that the voltage is moderately decreased after the insulator pin towards the insulator cap. While for contaminated insulators, the voltage distribution can be said gradually decreased from insulator pin towards the insulator cap as depicted in Figure 2 (b) to 2 (d). Similar finding was found in [20] where the potential distribution eventually shrinkage from $\mathrm{HV}$ electrode to the ground for one suspension glass insulator.

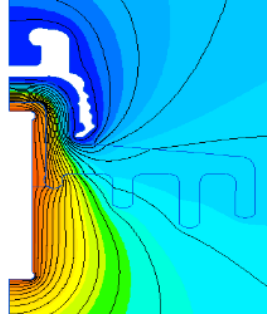

(a)

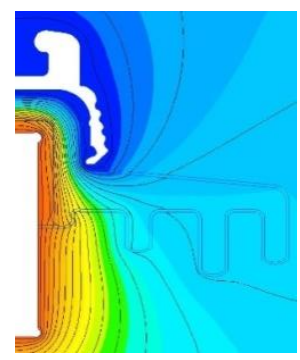

(c)

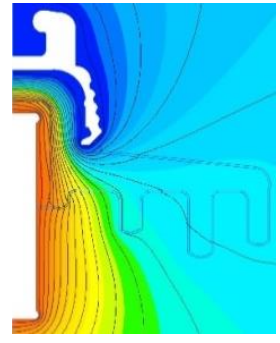

(b)

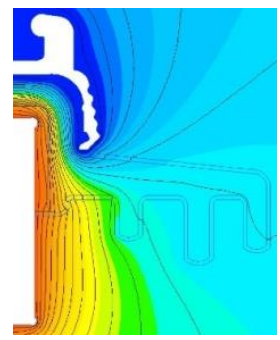

(d)

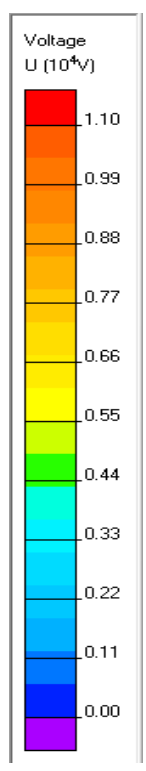

Figure 2. Voltage distribution contour plot of glass insulator for, (a) clean, (b) light, (c) medium, (d) heavy contamination

Apart from the voltage distribution, the distance of equipotential lines for clean and contaminated conditions also different. As can be seen from Figure 2, the equipotential lines become wider (represent in the circle) in the presence of contamination layer when compared to the clean insulator. This result shows that the voltage stresses are higher in the contamination area. 
The voltage distribution characteristic along the creepage distance for single glass insulator is demonstrated in Figure 3. It is noticed from the plot that the voltage is non-uniformly distributed along the creepage distance for clean insulator. However, the trend of distribution seems to become linear in the presence of contamination layer. The linearity distribution of voltage for contaminated insulators may be due to the conductivity created on the insulator surface enabling the leakage current (LC) flow and causing the voltage to increase [21]. However, in the real time operation services, the voltage distribution increased with the presence of contamination due to LC flow and formation of dry band.

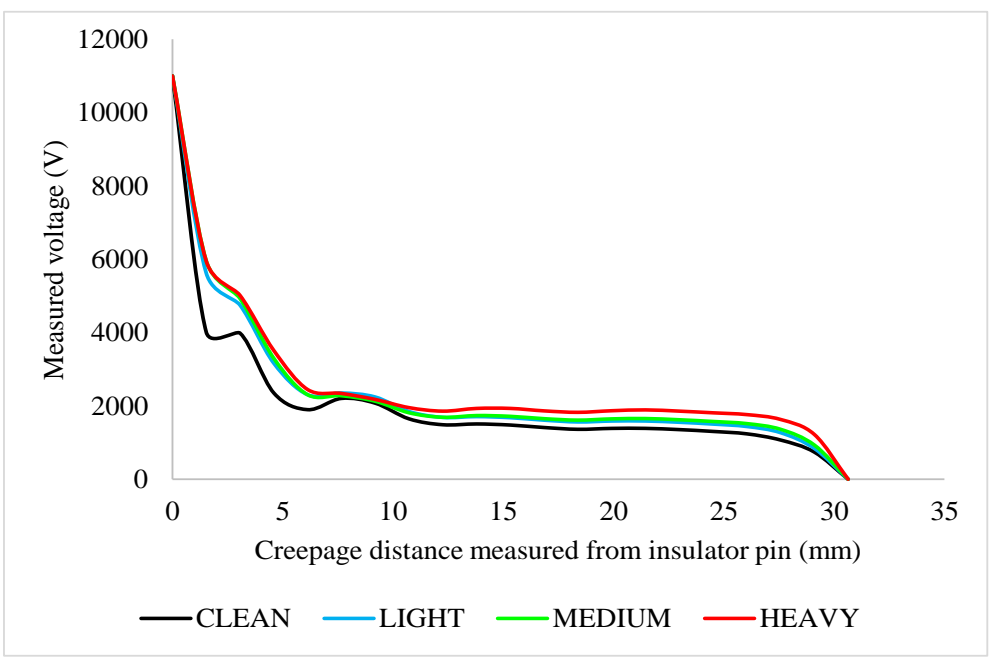

Figure 3. Voltage distribution characteristic along the creepage distance for single porcelain insulator

\subsection{Voltage distribution of single porcelain insulator}

The voltage distribution contour plot for a single porcelain insulator in clean and contaminated conditions is demonstrated in Figure 4. As can be seen in Figure 4, the distribution of voltage for porcelain insulator seems to have similar trend with glass insulator where the voltage was moderately decreased for clean and contaminated insulator from insulator pin to insulator cap. It is apparent that high potential was distributed more in the steel pin area for all the cases.

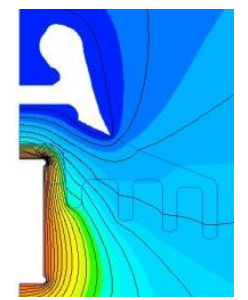

(a)

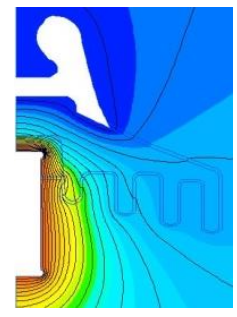

(c)

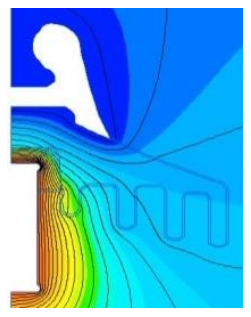

(b)

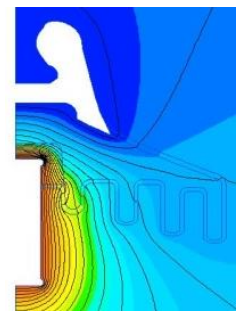

(d)

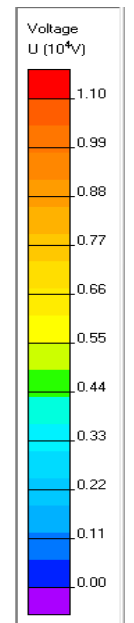

Figure 4: Voltage distribution contour plot of porcelain insulator for, (a) clean, (b) light, (c) medium, (d) heavy contamination 
Apart from that, the distance of equipotential lines (represent in the circle) for clean insulator is much wider compared to contaminated insulator. It is worth mentioning that the equipotential lines also play an important role in determining the condition of insulator in simulation study. The line width able to determine the level of voltage where wider equipotential lines represents lower voltage and vice versa. In this work, it is observed narrower equipotential lines is concentrated near to HV electrode while wider equipotential lines are focussed near to GND electrode.

Figure 5 depicts the distribution of voltage along the creepage distance for single porcelain insulator string. The results appear to indicate that the increasing of contamination layer on porcelain insulators surface has increased the voltage distribution of contaminated insulators. It is believed that larger thickness attract more trapped charges and allowed the creation of LC path on the insulator surface [22].

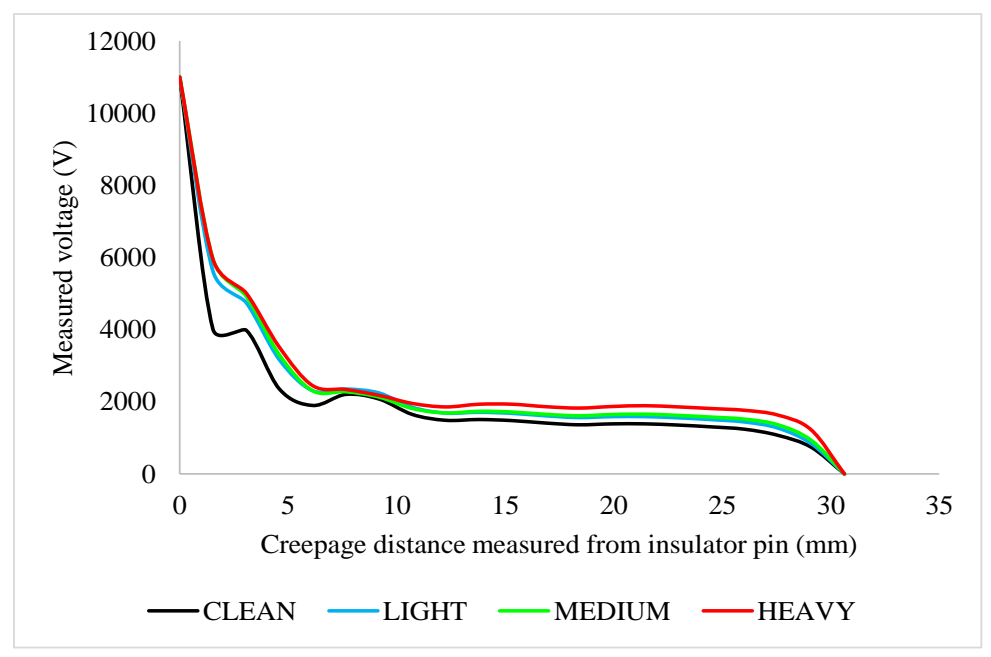

Figure 5. Voltage distribution characteristic along the creepage distance for single porcelain insulator

\subsection{Space charge distribution}

Space charge amplitude according to level of contamination for glass and porcelain insulators is depicted in Figure 6. What stands out in this figure is the dominance of space charge distribution in porcelain compared to glass insulators. However, both insulators type shows similar space charge distribution trend where the space charge amplitude is increased as the contamination level increased. It is important highlighting that the space charge distribution amplitude in Figure 6 is the average value. The space charge distribution studies can be considered as crucial since the presence of space charge at any region of insulator will distort the original electric field distribution and may result in the enhancement of local electric fields to the highest level over a long period of time [23-26].

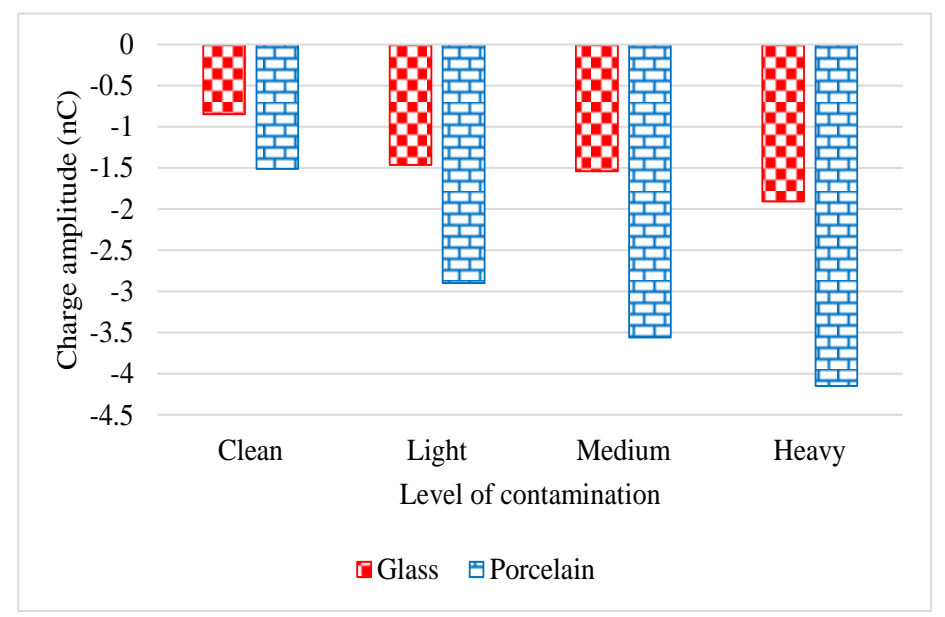

Figure 6. Space charge amplitude according to level of contamination for glass and porcelain insulators

Comparison on space charge and voltage distribution of high voltage insulator subjected... (N. A. Samuri) 


\subsection{Comparison between glass and porcelain string insulators on the effect of voltage distribution}

The comparison of voltage distribution for both glass and porcelain string insulator in clean and contaminated conditions is presented in Figure 7. It is proven that the glass insulator string gives higher value of voltage distribution compared to porcelain insulator string for all contamination levels.

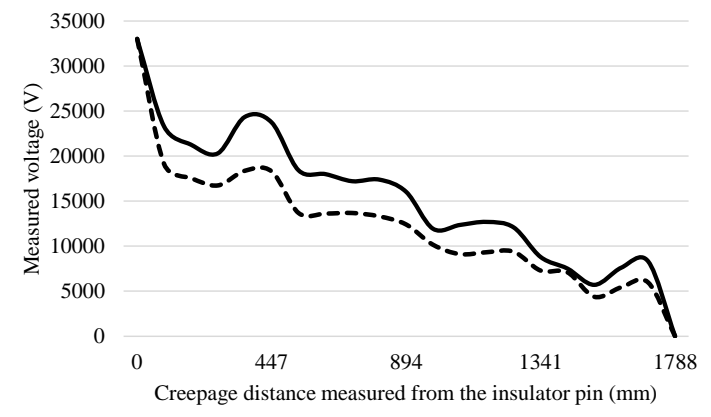

(a)

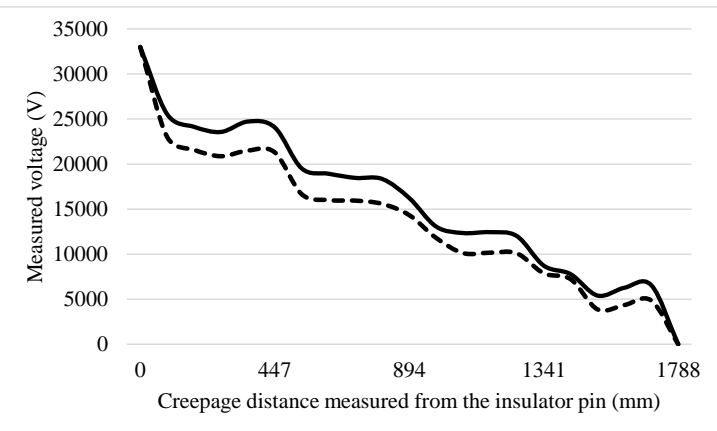

- Glass

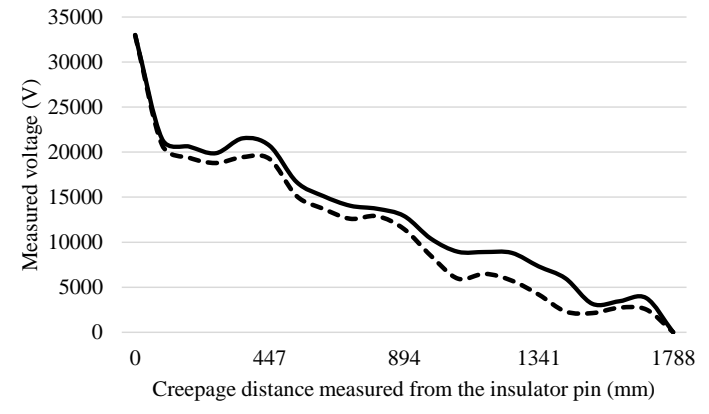

(b)

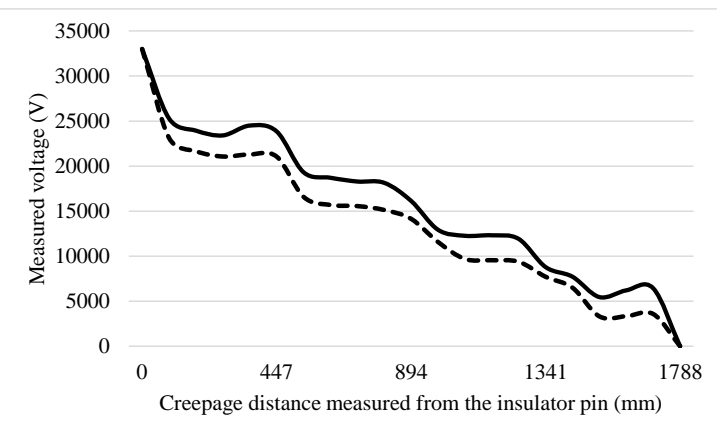

Porcelain

(d)

Figure 7. Voltage distribution comparison between glass and porcelain string insulator in, (a) clean, (b) light, (c) medium, (d) heavy contamination levels

\section{CONCLUSION}

The present article provides a contribution regarding the voltage and space charge distribution on a single unit and a string of four units glass and porcelain insulators under clean and contaminated conditions. The main findings of this work are highlighted according to their respective investigation conducted. Firstly, the high voltage string insulators with four units of glass and porcelain were simulated on voltage distribution, and it is shown that the higher chances of breakdown at glass insulator. As a result, the electric stress at glass insulator for all contamination levels are higher than porcelain insulator. Next, it is noticed in the voltage distribution along the string insulators is not linear for both porcelain and glass type. The highest voltage across each insulator happens at the insulator nearest to the high voltage line and exponentially decreases towards the insulator near to the ground. On top of that, when the level of contamination increases, the possibility of insulators to breakdown is higher. Lastly, the charge distribution for porcelain insulator is much higher than glass insulator.

\section{ACKNOWLEDGEMENTS}

The authors express their sincere gratitude to Research Management Centre Unversiti Tun Hussein Onn (UTHM) for awarding a research university grants (U863 and U948) as well as to Universiti Teknologi Malaysia (UTM), for the use of facilities and for awarding research university grant under vote $12 \mathrm{H} 07$ and 4F751 from Malaysia Ministry of Higher Education (MOHE). 


\section{REFERENCES}

[1] E. Lakervi and E. J. Holmes, Electricity Distribution Network Design: Institution of Engineering and Technology, 2003.

[2] M. S. Naidu and V. Kamaraju, High Voltage Engineering: McGraw Hill Education (India), 2013.

[3] W. A. Chisholm, "Insulator Leakage Distance Dimensioning in Areas of Winter Contamination using Cold-fog Test Results," IEEE Transactions on Dielectrics and Electrical Insulation, vol. 14, pp. 1455-1461, 2007.

[4] A. Kumada and S. Okabe, "Charge distribution measurement on a truncated cone spacer under DC voltage," IEEE Transactions on Dielectrics and Electrical Insulation, vol. 11, pp. 929-938, 2004.

[5] Z. Xu, "Space Charge Measurement and Analysis in Low Density Polyethylene," Ph.d. dissertation., University of Southampton., 2009.

[6] J. L. Davidson, T. J. Williams, and A. G. Bailey, "Electrostatic discharges between charged insulators and grounded spheres," Journal of Electrostatics, vol. 56, pp. 29-42, 2002.

[7] Y. Kishi, H. Miyake, Y. Tanaka, and T. Takada, "Relationship between breakdown and space charge formation in polyimide film under dc high stress," in 2009 IEEE Conference on Electrical Insulation and Dielectric Phenomena, 2009, pp. 146-149.

[8] W. Choo, G. Chen, and S. G. Swingler, "Space charge accumulation under effects of temperature gradient and applied voltage reversal on solid dielectric DC cable," in 2009 IEEE 9th International Conference on the Properties and Applications of Dielectric Materials, 2009, pp. 946-949.

[9] Y. Wang, J. Li, S. Wu, and P. Sun, "Influence of electrical aging on space charge dynamics of oil-impregnated paper insulation under AC-DC combined voltages," Journal of Electrical Engineering and Technology, vol. 8, pp. 1512-1519, 2013

[10] M. S. Khalil and B. S. Hansen, "Investigation of Space Charge in Low Density Polyethylene Using a Field Probe Technique," IEEE Trans. Electr. Insul., vol. 23, no 3, pp. 441-445, 1998.

[11] M. Ieda, "Study of Space Charge Effects in Polyethylene by Thermal-pulse Current Technique," vol. 25, no 3, pp. 509-514, 1990.

[12] S. Holé, T. Ditchi, and J. Lewiner, "Non-destructive methods for space charge distribution measurements. What are the differences?," IEEE Trans. Dielectr. Electr. Insul., vol. 10, no 4, pp. 670-677, 2003.

[13] M. P. Lalitha, K. V. P. Kumar, and V. Samala, "Modeling and simulation of porcelain disc insulators using finite element method in OPERA software," in 2014 Annual International Conference on Emerging Research Areas: Magnetics, Machines and Drives (AICERA/iCMMD), 2014, pp. 1-7.

[14] N. Othman, M. Piah, Z. Adzis, H. Ahmad, N. Ahmad, H. Kamarden, et al., "Characterization of charge distribution on the high voltage glass insulator string," Journal of Electrostatics, vol. 72, pp. 315-321, 2014.

[15] N. Othman, M. Piah, Z. Adzis, and H. Ahmad, "Measurement of surface charge distribution on glass insulator using steel mesh," in Power and Energy (PECon), 2014 IEEE International Conference on, 2014, pp. 105-108.

[16] S. A. Sediver, "Sediver toughened glass suspension insulators," ed, 2007.

[17] G. I. Corp, "Gamma Insulators," ed, 2018.

[18] V. Kontargyri, N. Ilia, I. Gonos, and I. Stathopulos, "Electric field and voltage distribution along insulators under pollution conditions," in 4th Mediterranean IEE Conference and Exhibition on Power Generation, Transmission, Distribution and Energy Conversion (Med Power 2004), 2004, pp. 15-17.

[19] M. El-Shahat and H. Anis, "Risk assessment of desert pollution on composite high voltage insulators," Journal of advanced research, vol. 5, pp. 569-576, 2014.

[20] S. Ilhan, A. Ozdemir, S. H. Jayaram, and E. A. Cherney, "Simulations of pollution and their effects on the electrical performance of glass suspension insulators," in 2012 Annual Report Conference on Electrical Insulation and Dielectric Phenomena, 2012, pp. 803-806.

[21] S. Moussavi, B. Sheikhdoragh, and A. Shayegani-Akmal, "Investigation on pollution factors on electric field and potential distribution of polymeric insulator," J. Basic. Appl. Sci. Res, vol. 2, pp. 12482-12491, 2012.

[22] A. Azizi Tousi and M. Mirzaie, The effect of severity and location of pollution on leakage current characteristics of porcelain insulators under different humidity condition vol. 13, 2013.

[23] K. Ueno, S. Nakamura, H. Shimotani, A. Ohtomo, N. Kimura, T. Nojima, et al., "Electric-field-induced superconductivity in an insulator," Nature materials, vol. 7, p. 855, 2008.

[24] A. See, J. C. Fothergill, L. A. Dissado, and J. M. Alison, "Measurement of space-charge distributions in solid insulators under rapidly varying voltage using the high-voltage, high-speed pulsed electro-acoustic (PEA) apparatus," Inst. Phys. Publ. Meas. Sci. Technol., vol. 12, pp. 1227-1234, 2001.

[25] I. W. McAllister, G. C. Crichton, and A. Pedersen, "Charge Accumulation in DC Cables: A Macroscopic Approach," Conf. Rec. 1994 IEEE Int. Symp. Electr. Insul. Pittsburgh, PA USA, pp. 212-216, 1994.

[26] A. E. Davies and G. Chen, "Electric Stress Distribution in Polymeric Insulation Containing Defect Sites and Space Charge," COMPEL - Int. J. Comput. Math. Electr. Electron. Eng., vol. 11, no. 1, pp. 237-240, 1992. 


\section{BIOGRAPHIES OF AUTHORS}
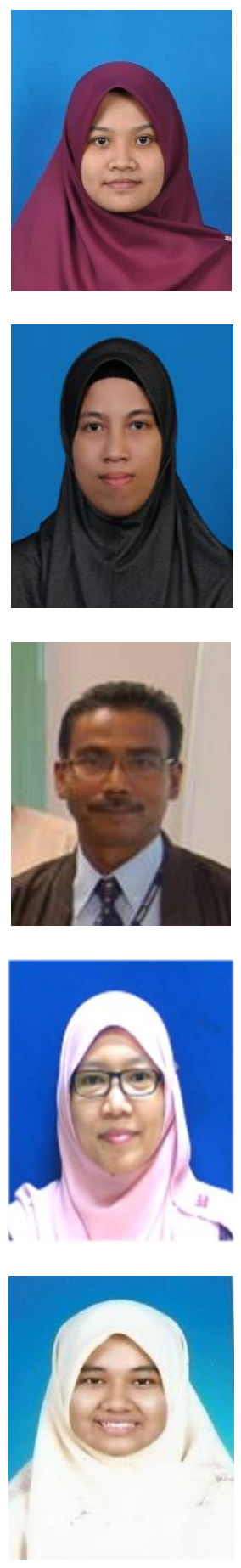

Nurul Aini Samuri-was born in Batu Pahat, Johor, Malaysia on February 24, 1993. She went to SMK Tun Sardon and complete her high school at 2010. After high school, she continued her study and received both the Diploma of Electrical Engineering and Bachelor of Electrical Engineering (Hon.) from University Tun Hussein Onn Malaysia (UTHM), Johor in 2014 and 2017 respectively. Her main area of research interest is space charge distribution on HV insulator under different level of contamination

Nordiana Azlin Othman was born in Johor, Malaysia on January 19, 1986. She is currently a Lecturer at the Department of Electrical Power Engineering, Universiti Tun Hussein Onn Malaysia. She received B. Eng. in Electrical Engineering from Universiti Teknologi Malaysia (UTM) in 2010 and PhD in High Voltage Engineering from UTM in 2016. Her research interest includes the detection and diagnostics of partial discharge and space charge in insulation for condition monitoring.

Mohamed Afendi Mohamed Piah was born in Taiping, Perak, on November 8, 1963. He is an associate professor at Faculty of Electrical Engineering, Universiti Teknologi Malaysia (UTM) and a fellow member of the Institute of High Voltage and High Current (IVAT). He received the B.Elect. Eng. degree from UTM in 1986, M.Sc in Power System from University of Strathclyde, UK in 1990 and PhD in High Voltage Engineering from UTM in 2004. His research interests include high voltage insulation diagnostic and co-ordination, electrical discharges, polymer nanocomposites insulating materials and insulator condition monitoring.

Nor Akmal Mohd Jamail is a lecturer of Universiti Tun Hussein Onn Malaysia since 2008. She received the Bachelor degree in Electrical Engineering from Universiti Teknologi Malaysia in 2005, M.Eng in Power System from Universiti Tun Hussein Onn Malaysia in 2007 and Ph.D. in High Voltage Engineering from Universiti Teknologi Malaysia, in 2015. Her research interests include condition monitoring of polymer nanocomposite insulation for HV purpose, dielectrics and electrical insulation. She is registered with the Board of Engineers Malaysia (BEM).

Hanan Rosli was born and raised in Sungai Petani, Kedah, Malaysia on January 24, 1994. After completing high school in MARA Junior Science College (MJSC) Kepala Batas (2011), she went to Kedah Matriculation College for a year and pursue her study in Bachelor of Electrical Engineering (Hons) in University Tun Hussein Onn Malaysia (UTHM), Johor (2017). Her research interest is more focus in high voltage insulation field. 